\title{
Prototipo para el análisis del crecimiento vehicular y de estaciones de monitoreo atmosférico
}

\section{Prototype for the analysis of vehicle growth and atmospheric monitoring stations}

ROMO-GONZALEZ, Ana Eugenia†* \& VILLALOBOS-ALONZO, María de los Ángeles

Universidad Tecnológica de Jalisco

ID $1^{\mathrm{er}}$ Autor: Ana Eugenia, Romo-Gonzalez / ORC ID: 0000-0002-4653-2593, CVU CONACYT ID: 212291

ID $1^{\mathrm{er}}$ Coautor: María De Los Ángeles, Villalobos-Alonzo / ORC ID: 0000-0003-3052-8271, CVU CONACYT ID: 212718

DOI: $10.35429 /$ JTEN.2020.14.4.13.19

Recibido 03 de Junio, 2020; Aceptado 30 Octubre, 2020

\section{Resumen}

Coexiste un reconocimiento universal de la importancia y necesidad de establecer acciones climáticas más ambiciosas y efectivas en todos los sectores involucrados; gobierno, sector privado y sociedad con el objetivo de actuar en conjunto para disminuir y frenar el aumento de la temperatura global en el presente siglo y con ello, evitar efectos devastadores e irremediables en el cambio climático. En este sentido, la implementación de sistemas de monitoreo atmosférico ha permitido identificar las emisiones de partículas y gases contaminantes relacionadas directamente con los vehículos de motores a gasolina y diesel; por lo tanto el objetivo del este proyecto consiste en el diseño de un prototipo de sistema para dispositivos móviles con sistema operativo Android, que permita proyectar el aumento o disminución de gases contaminantes empleando los datos estadísticos de crecimiento vehicular y de las estaciones de monitoreo atmosférico del estado de Jalisco, México, por lo que constituye una herramienta tecnológica valiosa cuya contribución principal se enfoca en mantener a la población informada acerca de la importancia de la disminución de contaminantes que puedan deteriorar la salud.

\section{Prototipo, Crecimiento vehicular, Monitoreo} atmosférico

\begin{abstract}
There is a universal recognition of the importance and need to establish more ambitious and effective climate actions in all the sectors involved; government, private sector and society with the aim of acting together to decrease and stop the increase in global temperature in the present century and thereby avoid devastating and irremediable effects on climate change. In this sense, the implementation of atmospheric monitoring systems has made it possible to identify the emissions of particulate and polluting gases directly related to gasoline and diesel engine vehicles; therefore, the objective of this project is to design a prototype system for mobile devices with the Android operating system, which allows projecting the increase or decrease of polluting gases using statistical data on vehicle growth and atmospheric monitoring stations in the State of Jalisco, Mexico, therefore it constitutes a valuable technological tool whose main contribution focuses on keeping the population informed about the importance of the reduction of contaminants that can deteriorate health.
\end{abstract}

Prototype, Vehicle growth, Atmospheric monitoring

Citación: ROMO-GONZALEZ, Ana Eugenia \& VILLALOBOS-ALONZO, María de los Ángeles. Prototipo para el análisis del crecimiento vehicular y de estaciones de monitoreo atmosférico. Revista de Ingeniería Tecnológica. 2020. 4-14: 13-19

\footnotetext{
* Correspondencia del Autor (Correo electrónico: aromo@utj.edu.mx)

$\dagger$ Investigador contribuyendo como primer autor.
} 


\section{Introducción}

El cambio climático se ha convertido en un tema prioritario de la agenda de organismos internacionales, que ha impactado en dimensiones políticas, sociales y culturales, orientando a los países y gobiernos a desplegar estrategias para frenar el calentamiento global. Entre los desafíos a nivel mundial, tanto en Latinoamérica como en países del Caribe, se encuentra la descarbonización de la economía como nueva alternativa del desarrollo sostenible, "con la intensión de reducir las emisiones en más de un $80 \%$ para el 2050 y limitar a un $2^{\circ} \mathrm{C}$ el aumento de la temperatura del planeta," (Marco de las Naciones Unidas sobre cambio climático (COP 20), 2014).

El monitoreo de la información de gases contaminantes a través de aplicaciones que muestren el análisis sintetizado de los datos es importante ya que de acuerdo con el informe del Instituto de Desarrollo Sostenible y Relaciones Internacionales, (2015:4), que lidera "The Deep Decarbonization Pathways Project" (DDPP) donde colaboran 16 países, coinciden en la "descarbonización profunda" en la economía y el establecimiento de una trayectoria a largo plazo para que los resultados esperados sean satisfactorios y consistentes en le reducción de emisiones netas per cápita de $\mathrm{CO} 2$ para el año 2050 .

La propuesta de reducción de emisiones intenta limitar el uso de combustibles fósiles y otros emisores de dióxido de carbono (CO2) de los cuales, estos 16 países son responsables de más del 70\% de las emisiones globales de GEI (Gases de efecto invernadero). Figura 1.

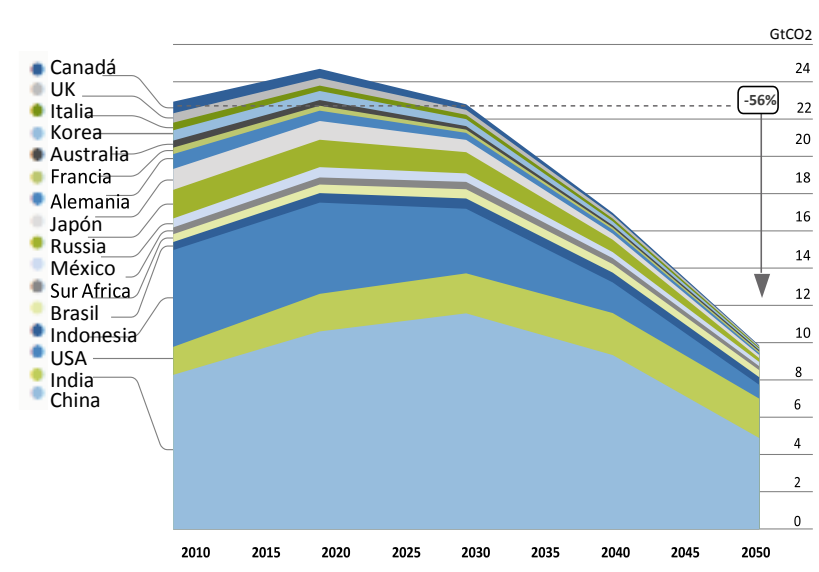

Figura 1 Trayectorias de emisiones de energía $\mathrm{CO} 2$, 2010-2050, mostrando los escenarios de reducción más ambiciosos para todos los países DDPP

Fuente: DDPP, (2015: 4)
"La proyección de la descarbonización al 2050 se vuelve factible en un escenario ambicioso donde se pretende la reducción al $57 \%$ de las trayectorias de emisiones de energía $\mathrm{CO} 2$, considerando un crecimiento poblacional de un $17 \%$ y un PIB de $250 \%$ correspondiente al promedio acumulado del $3.1 \%$ en 40 años" (Deep Decarbonization Pathways Project, 2015: 4).

En el caso de México, se alinea a la intensión de la reducción de emisiones consistentes con el objetivo $2^{\circ} \mathrm{C}$ acordado en el DDPP en tres pilares de descarbonización profunda:

1. Eficiencia y ahorro energético,

2. Electricidad baja en carbono, y

3. Sustitución de combustibles.

Su situación al 2010 es de "493,450.63 miles de toneladas de C02" (SEMARNAT, 2012), "las emisiones de GEI están acrecentando al 2015 debido al aumento del consumo de combustibles fósiles" (Tovilla et al., 2015: 2).

El uso de la energía se extendió sobre los siguientes combustibles: "gasolina (32\%), electricidad (16\%), Diesel (16\%), gas natural (11\%), GLP (10\%) y madera (5\%). Aproximadamente el $30 \%$ de toda la energía utilizada actualmente en México está dedicado al transporte, y cerca del $70 \%$ de esa energía es demandada por los vehículos particulares.

Estas cifras reflejan el aumento del vehículo de particular que se duplicó de 2000 a 2010 a aproximadamente 207 vehículos por cada mil habitantes, y en las emisiones de GEI del transporte creció a una tasa anual del 2,9\% entre 1990 y 2010" (Tovilla et al., 2015: 12).

De acuerdo con Pathways to deep decarbonization in Mexico, (2015:8) "las emisiones totales de GEI alcanzaron $694 \mathrm{MtCO} 2$ en 2010 (Figura 2). 


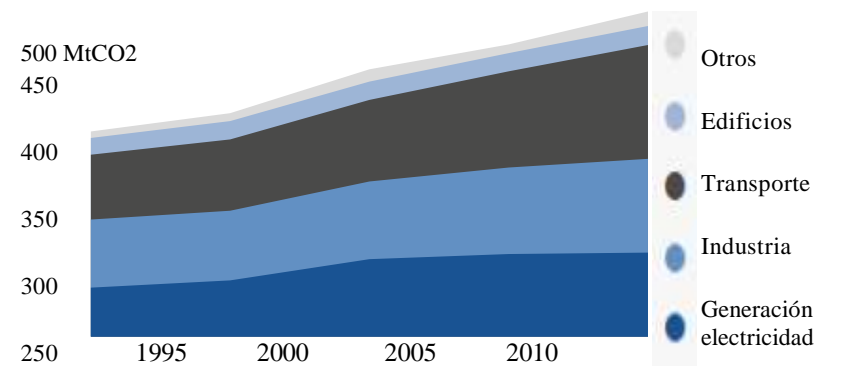

Figura 2 Histórico de emisiones de $\mathrm{CO} 2$ relacionadas con la energía en México por sector. *Cifras representados en millones

Fuente: Tovilla, (2015: 12)

En la Figura 1 se observa que alrededor del $60 \%$ de ellas corresponden a las emisiones de $\mathrm{CO} 2$ derivadas de la combustión de combustibles fósiles para usos energéticos (424 Mton)".

Por lo tanto, la mayor fuente de emisiones de GEI en México se originan por el uso del transporte $(23 \%$ de todas las emisiones de GEI en 2010) y la generación de electricidad $(17 \%)$.

El parque vehicular es uno de los factores de las emisiones del trasporte, ya que aunque el crecimiento de la población en México se ha mantenido estable, no ha sido así el de vehículos particulares porque del 2000 al 2018 hemos tenido un crecimiento del $68 \%$ (Figura 3)

Crecimiento Poblacional en \%

Periodo (2000-2018)

\section{贯 $\left.||||||||||||||||||||\right|^{m \times}$}

Crecimiento Vehicular en $\%$

Periodo(2000-2018)

\section{Bी||||||||||||||||| ${ }^{\text {का }}$}

Figura 3 Relación de la cantidad de vehículos respecto a los habitantes en México al 2018

Fuente: Elaboración propia con datos estadísticos de INEGI, (2018)

En el año 2000, México contaba con 97 millones de habitantes y 9.78 millones de autos; 10 habitantes por vehículo. Sin embargo, en 2015, la población creció a 119.5 millones y los automóviles a 26.5 millones, reduciéndose a 4.5 habitantes por vehículo.
En 2018 existían registrados en INEGI 31'214,090 millones los automóviles por 132' 865,407 millones de habitantes alcanzando un promedio de 4.2 habitantes por vehículo, aumentando en un $45 \%$ las emisiones de GEI por transporte particular.

Las ciudades de México con mayor índice de parque vehicular y de emisiones de $\mathrm{CO} 2$ derivadas de los combustibles fósiles para usos energéticos se presentan en la Tabla 1.

\begin{tabular}{|l|r|r|r|}
\hline \multicolumn{1}{|c}{ Ciudad Habitantes } & \multicolumn{1}{c|}{$\begin{array}{c}\text { Autos } \\
\text { particulares }\end{array}$} & \multicolumn{1}{c|}{$\begin{array}{c}\text { Habitantes } \\
\text { por } \\
\text { vehículo }\end{array}$} \\
\hline $\begin{array}{l}\text { Estado de } \\
\text { México }\end{array}$ & 16.1 & 7.3 & 2.2 \\
\hline $\begin{array}{l}\text { Cd. de } \\
\text { México } \\
\text { (CDMX). }\end{array}$ & 8.9 & 5.4 & 1.6 \\
\hline Jalisco & 7.8 & 3.6 & 2 \\
\hline
\end{tabular}

Tabla 1 Ciudades de México con mayor emisión de CO2 por vehículos particulares

Fuente: Elaboración propia con datos estadísticos de INEGI

Como se identifica en la Tabla 1, la CDMX cuenta con alto parque vehicular particular con respecto a la tasa poblacional aproximada.

Las emisiones de GEI deben ser medidas de manera constante por lo que en México la SEMARNAT despliega una red de monitorio con 33 estaciones distribuidas en el territorio nacional, para evaluar el estado de la calidad del aire con respecto a la concentración de los contaminantes criterio (Partículas suspendidas PM10 y PM2.5, Ozono (03), Dióxido de azufre (SO2), Dióxido de nitrógeno (NO”) y Monóxido de carbono $(\mathrm{Co})$.

El último inventario Nacional de gases de efecto invernadero (INECC, 2015) (Figura 4), que emite el Transporte es el $25.1 \%$ de las partículas contaminantes y gases (Bióxido de Carbono (CO2) $71.1 \%$ y Metano en un $20.8 \%$. En el estado de Jalisco, existe un Sistema de monitoreo atmosférico compuesto por 10 estaciones fijas y una móvil que opera con el sistema WINAQMS. 


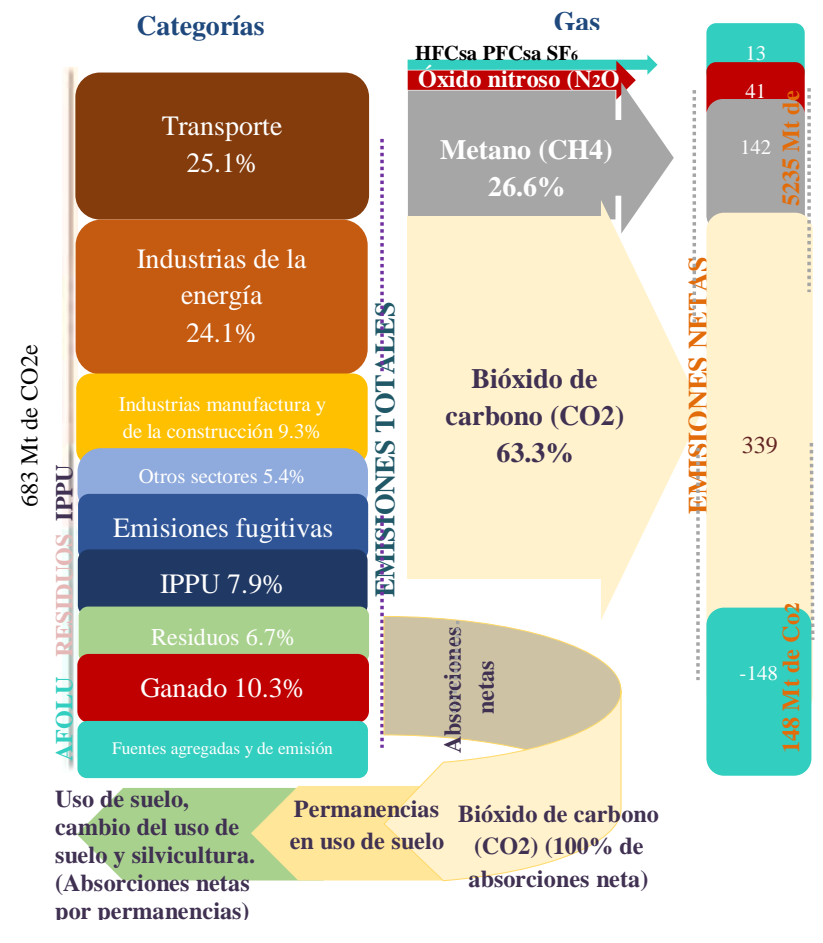

Figura 4 (Edición) Inventario nacional de emisiones de gases y compuestos de efecto invernadero GEI.

Fuente: INECC y SEMARNAT, 2018

En el Marco de DDPP, México busca implementar una agenda de acción 2015-2020 Generar visión integral de descarbonización de los sistemas de generación y uso de energía (Eficiencia / Generación de cero emisiones / Sistemas de transporte), 2020-2025 Programa de acción intersectorial, con rutas acordadas y marco institucional correspondiente (Planeación / Marco regulatorio e institucional / Instrumentos económicos y de política / Recursos / Métricas y monitoreo) e instrumentar el cambio siguiendo rutas acordadas de inversión y tecnologías.

Las estaciones de monitoreo para la medición del aire en la zona metropolitana de Guadalajara, en el estado de Jalisco realizan un muestro con intervalos de una hora en un radio de cobertura de $2 \mathrm{~km}$.

Por lo tanto, aunque la información de las partículas contaminantes está disponible para la consulta de la población, a través de la descarga de archivos de diversos tipos, el promedio de los ciudadanos además de desconocer la existencia de los datos carece de las habilidades necesarias para su interpretación. En este contexto, el desarrollo de herramientas en plataformas de alta penetración como los dispositivos móviles es pertinente debido a que le permite al sector social mantenerse informado sobre los efectos de las mediadas de descarbonización y el reto de la disminución de emisiones de $\mathrm{CO} 2$ en el país.
Por lo que el objetivo del presente trabajo consiste en diseñar un sistema para dispositivos móviles que permita proyectar el aumento o disminución de gases contaminantes empleando los datos estadísticos de crecimiento vehicular y de las estaciones de monitoreo atmosférico del estado de Jalisco, México, por lo que constituye una herramienta tecnológica valiosa para mantener a la población informada.

Para abordar el diseño del prototipo, en este artículo se presenta, en el apartado de metodología el desglose del método utilizado y los pasos realizados para el desarrollo. Posteriormente se presentan los resultados respecto a la implementación propuesta y finalmente las conclusiones.

\section{Metodología}

En este trabajo se utiliza el método ágil (Rahimian y Ramsin, 2008) para el desarrollo de proyectos en el área de software con enfoque en tecnología aplicada. Se presentan de forma esquemática (Figura 5) las tres fases que conforman la metodología:

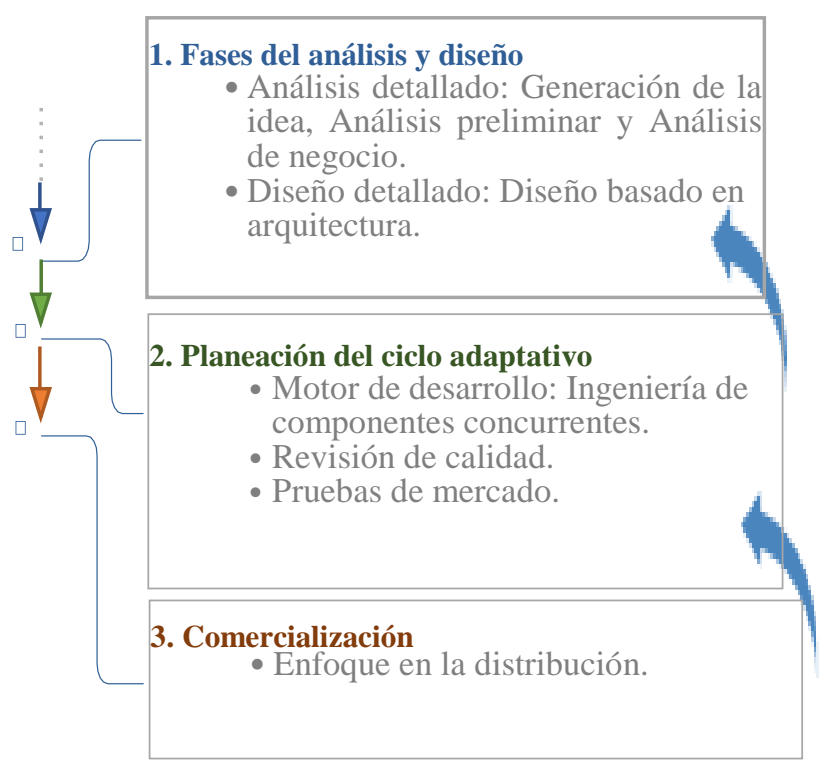

Figura 5 Fases de la metodología ágil (Rahimian y Ramsin, 2008)

En la etapa de análisis detallado se identifica como eje central del proyecto (idea) el análisis proyectado de los contaminantes identificados por las diez estaciones de monitoreo atmosférico y una unidad móvil del estado de Jalisco, México; así como los datos estadísticos del crecimiento vehicular, principal emisor de gases contaminantes. 
El análisis preliminar y de negocio se basa en la alta penetración de dispositivos móviles y teléfonos inteligentes en México así como de la plataforma operativa más utilizada en América latina (Romo-González et al., 2017).

En el diseño basado en la arquitectura se consideran las limitaciones de los desarrollos en los dispositivos móviles y los mecanismos de conexión para la descarga de documentos estadísticos en diversos formatos (Excel, pdf). De los formatos se extraen los campos relevantes con los que se diseña la base de datos para el sistema. Para el cálculo de las proyecciones del crecimiento vehicular se utiliza la fórmula de Tránsito Promedio Diario Anual (TPDA) con el que se obtienen los volúmenes de transito fututo (Grisales, 2007).

Finalmente, se realizan las tablas comparativas de los gases contaminantes emitidos a la atmósfera y que son campados por las estaciones de monitoreo atmosférico contra el crecimiento del parque vehicular.

\section{Resultados}

De acuerdo la metodología establecida, como primer paso se identificaron las partículas contaminantes con registro en las estaciones climatológicas. Desde el 01 de enero 1996, cada una de las diez estaciones de monitoreo atmosférico que componen el Sistema de medición del aire en la zona metropolitana de Guadalajara Jalisco realizan un muestro con intervalos de una hora y tienen un radio de cobertura de $2 \mathrm{~km}$. Los diez compuestos que se identifican se presentan en la tabla 2 :

\begin{tabular}{|c|c|c|}
\hline No. & Emisión & Observación \\
\hline 1 & $\mathrm{CO}$ & \multirow{10}{*}{$\begin{array}{l}\text { Los gases que emiten los motores de } \\
\text { gasolina y que son considerados } \\
\text { como contaminantes son: monóxido } \\
\text { de carbono (CO), hidrocarburos no } \\
\text { quemados (HC), óxidos de nitrógeno } \\
\text { (NOx), óxidos de azufre (SOx) y } \\
\text { dióxido de carbono o CO2. } \\
\text { Los motores de diesel: NOx, HC, CO, } \\
\text { dióxido de carbono, dióxido de } \\
\text { azufre (Cox), y aldehídos y } \\
\text { micropartículas de hollín (Mp) } \\
\text { procedentes del carbón. }\end{array}$} \\
\hline 2 & NO2 & \\
\hline 3 & NOX & \\
\hline 4 & $\mathrm{O} 3$ & \\
\hline 5 & PM10 & \\
\hline 6 & $\mathrm{RH}$ & \\
\hline 7 & $\mathrm{SO} 2$ & \\
\hline 8 & TMP & \\
\hline 9 & WDR & \\
\hline 10 & WSP & \\
\hline
\end{tabular}

Tabla 2 Listado de emisiones contaminantes disponibles en los registros emitidos por las diez estaciones de monitoreo atmosférico y una unidad móvil del estado de Jalisco, México

Fuente: Elaboración propia con datos de las estaciones de monitoreo
A partir de la Tabla 1 se establece que las emisiones directamente relacionadas con los vehículos son CO, NOX y TMP. El incremento de estas partículas se puede apreciar en la Figura 6.

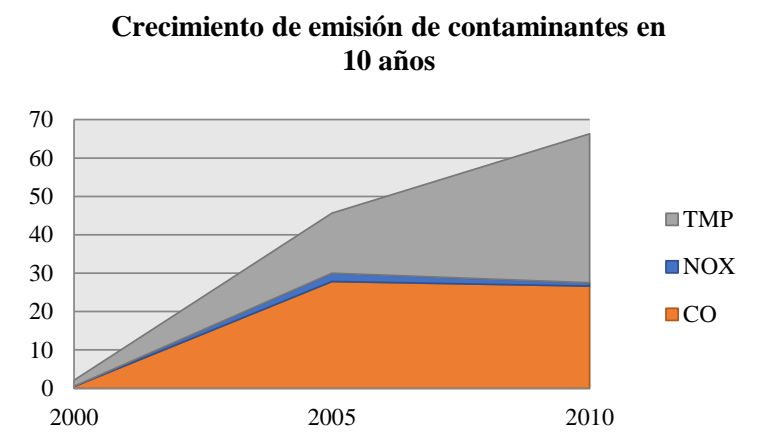

Figura 6 Crecimiento de contaminantes en el estado de Jalisco en un periodo de 10 años

Fuente: Elaboración propia a partir del análisis de datos de las estaciones de monitoreo atmosférico (SIMAJ, 2018)

Posteriormente se recupera la información del crecimiento vehicular para el estado. Con respecto a este indicador los datos encontrados muestran que en casi 4 décadas se ha incrementado en un $642.7 \%$, lo que ha contribuido de manera significativa en el aumento de emisión de contaminantes a la atmósfera. En la Figura 7 se muestra el crecimiento desde el año 1980 al 2016.

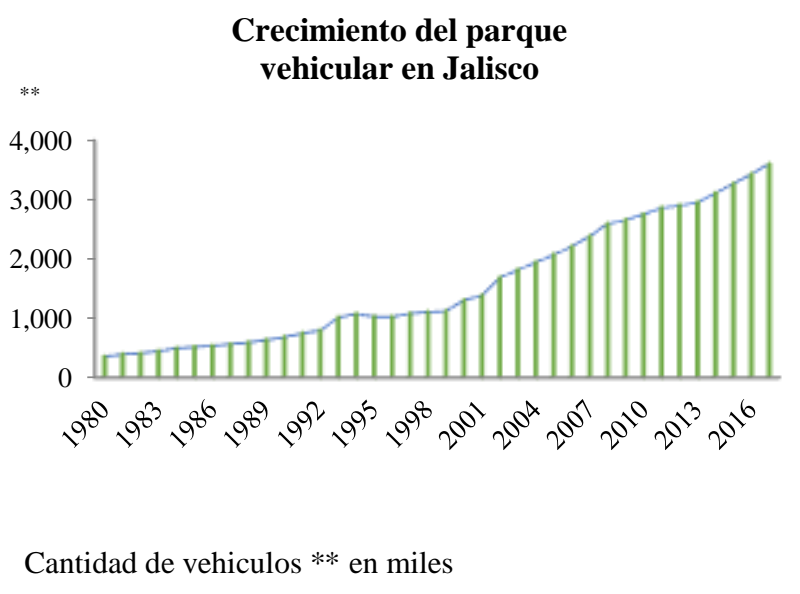

Figura 7 Crecimiento del parque vehicular en el estado de Jalisco

Fuente: Elaboración propia según datos de INEGI, (2017)

La información del parque vehicular se contrasta con el crecimiento poblacional para determinar la relación de vehículos por habitante (Figura 8). 
Tendencia de vehiculos por habitante

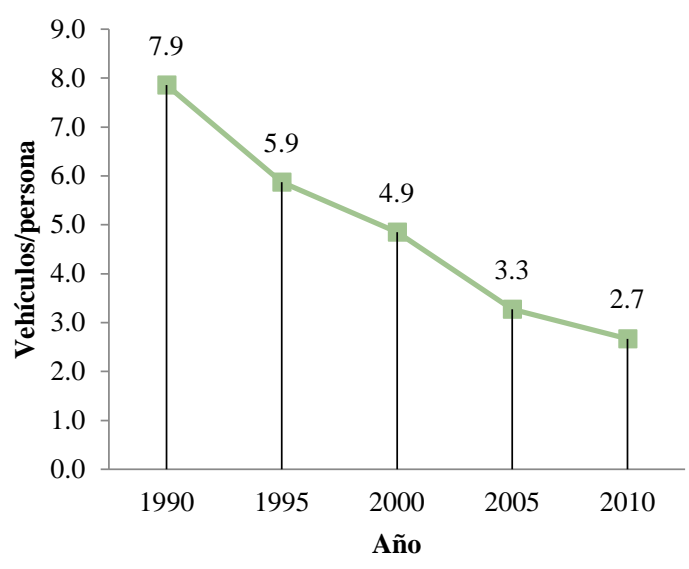

Figura 8 Relación del crecimiento del parque vehicular respecto a la cantidad de habitantes en el estado de Jalisco Fuente: Elaboración propia según datos de INEGI, (2017)

La construcción de la Tabla 2 y de las Figuras 6 y 7 se sustenta en la sustracción y posterior análisis de datos masivos de diversas fuentes. Esta estructura permitió esquematizar la arquitectura para el diseño del prototipo presentado en la Figura 9.

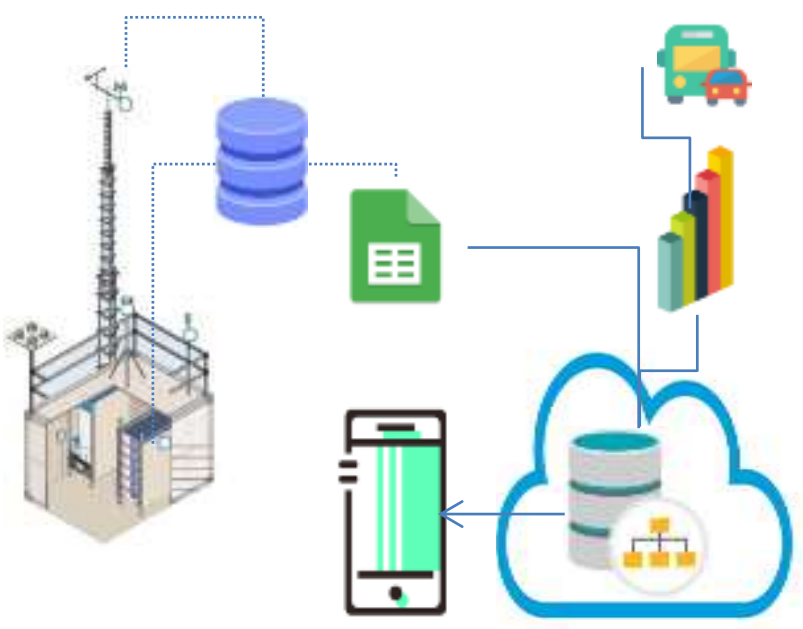

Figura 9 Arquitectura del prototipo de para el análisis del crecimiento vehicular y de estaciones de monitoreo atmosférico

Fuente: Elaboración Propia

El esquema de la Arquitectura propuesto (Figura 8) contiene la recolección masiva de datos generados por las estaciones de monitoreo atmosférico y su descarga en formatos de hojas electrónicas a los que se integran la información del parque vehicular y el crecimiento poblacional. Los datos y se análisis se conjuntan en una base de datos almacenada en la nube.
Una vez concluida la etapa desarrollo de la base de datos para el prototipo, se desarrolló el prototipo de la aplicación móvil con la estructura presentada en la Figura 10.

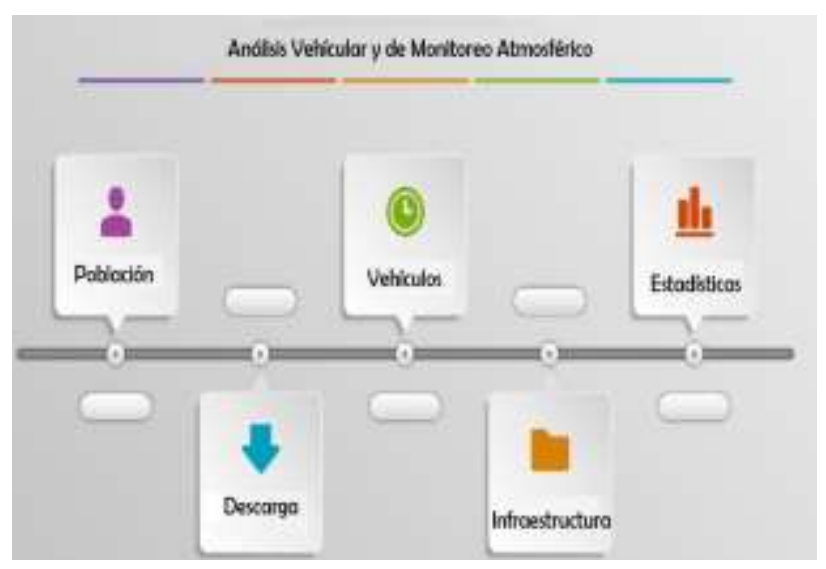

Figura 10 Interfaz del prototipo de para el análisis del crecimiento vehicular y de estaciones de monitoreo atmosférico

Fuente: Elaboración Propia

Los apartados relativos a la población y vehículos muestran el crecimiento poblacional y el del parque vehicular por periodos que pueden ser seleccionados por el usuario.

En las estadísticas se pueden mostrar gráficas como las de la Figura 7 además de las tendencias de emisiones de partículas a la atmosfera en períodos específicos.

Las gráficas pueden descargarse en formato de imagen desde el apartado de descargas. Además de la infraestructura sobre el monitoreo ambiental y su alcance de acuerdo con la zona de ubicación, como se muestra en la Figura 11.

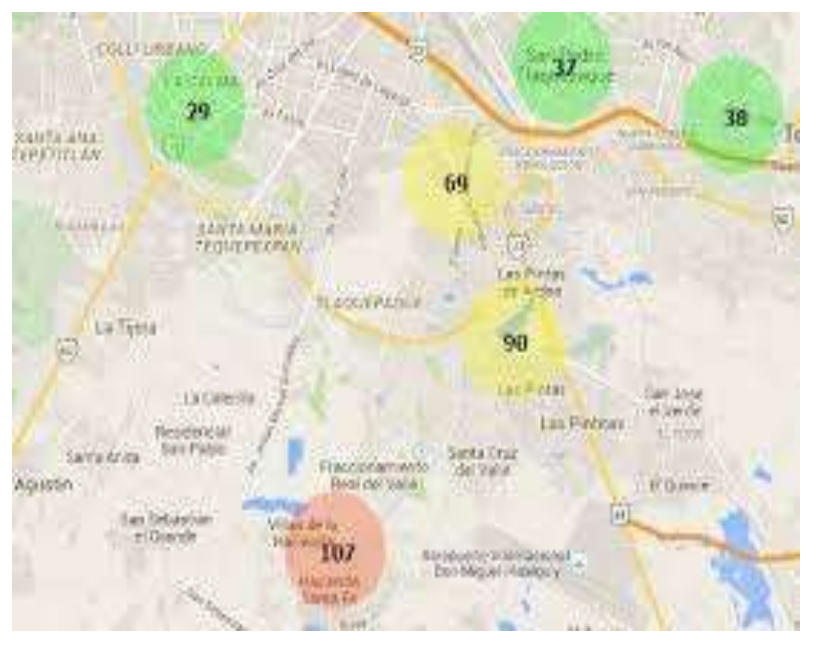

Figura 11 Especificación de alcance de las estaciones de monitoreo atmosférico

Fuente: SIMAJ (2018) 
A partir del diseño del prototipo se comienza el desarrollo para dispositivos móviles que cuentan con el sistema operativo Android debido a la alta penetración, lo que permite acercar la información a un sector más amplio de la población.

\section{Conclusiones}

Aunque el nivel de contaminación antropogénica identificada en la zona metropolitana de Guadalajara Jalisco se encuentra a disposición de la sociedad a través de archivos con diversos formatos, no todos los ciudadanos poseen la capacidad para realizar un análisis de los datos. Por lo tanto el desarrollo de un sistema que presente la información integrada constituye una herramienta para acercar al sector social a la problemática de los niveles de contaminación y su influencia en el cambio climático con impactos negativos.

La extensión de la aplicación del prototipo a otros estados de la República es factible a partir de su desarrollo incremental mediante menú de selección de estados e identificación de estaciones de monitoreo atmosférico por entidad.

\section{Referencias}

Deep Decarbonization Pathways Project (2015). Pathways to deep decarbonization 2015 report executive summary, SDSN - IDDRI. Consultado 14 de octubre de 2018. En: http://unsdsn.org/wpcontent/uploads/2015/09/DDPP_EXESUM.pdf

Grisales, J. C. (2007). El volumen de tránsito atraído y desarrollado en carreteras de Colombia. Un caso real. Revista de Topografía AZIMUT, 1, 41-52.

Instituto Nacional de Ecología y Cambio Climático (INECC) y Secretaría de Medio Ambiente y Recursos Naturales (SEMARNAT), 2018. México: Inventario Nacional de Emisiones de Gases y Compuesto de Efecto Invernadero 1990-2015, se produjo con financiamiento del [Fondo para el Medio Ambiente Mundial (FMAM)], implementado por [el Programa de Naciones Unidas para el Desarrollo (PNUD). En: http://cambioclimatico.gob.mx:8080/xmlui/han dle/publicaciones/226

INEGI. (2018). Parque vehicular. Consultado el 14 de oct. de 18. En: http://www.beta.inegi.org.mx/temas/vehiculos/
INEGI. (2017) Vehículos de motor registrados en circulación. Consulta interactiva. Disponible:http://www.inegi.org.mx/est/conteni dos/proyectos/registros/economicas/vehiculos/d escripciones.aspx\#

Ministerio de Ambiente y Desarrollo Sostenible. Acuerdo París. Consultado el 13 de octubre de 2018.

En: http://www.minambiente.gov.co/index.php/asu ntos-ambientales-sectorial-y-urbana

Observatorio ambiental Bogotá. (2018). Parque vehicular. Consultado el 13 de octubre de 2015. En:

http://oab.ambientebogota.gov.co/es/documenta cion-e-investigaciones

ONU. (2015). Conferencia Mundial de Cambio Climático COP 20. (Documento web). Consultado 13 de octubre de 2018. En: http://onu.org.pe/wpcontent/uploads/2014/07/Triptico-COP-20.pdf

Rahimian, V., \& Ramsin, R. (2008, June). Designing an agile methodology for mobile software development: A hybrid method engineering approach. In Research Challenges in Information Science, 2008. RCIS 2008. Second International Conference on (pp. 337342). IEEE.

Romo-González, A., Villalobos-Alonzo, M, Avila- Garcia, D., Marquez-Sanchez, M. (2017). "Aplicación móvil para un sistema de diagnóstico en procesos de investigación, desarrollo tecnológico e innovación". Revista de Tecnologías de la Información, Volume 4 Number 12. ISSN: 2410-4000, pages 25-36.

Semarnat. (2012), Elaboración propia con base en: Coordinación del Programa de Cambio Climático y Consejo Nacional de Población (Conapo). Proyecciones de la población de México 2010-2050 y estimaciones 19902009. Abril. 2013. México.

SIMAJ (2018). Sistema de monitoreo atmosférico de Jalisco. Secretaria de medio ambiente y desarrollo territorial http://siga.jalisco.gob.mx/aire/Infra.html

Tovilla, J. et al. (2015). Pathways to deep decarbonization in Mexico, SDSN - IDDRI.

ROMO-GONZALEZ, Ana Eugenia \& VILLALOBOS-ALONZO, María de los Ángeles. Prototipo para el análisis del crecimiento vehicular y de estaciones de monitoreo atmosférico. Revista de Ingeniería Tecnológica. 